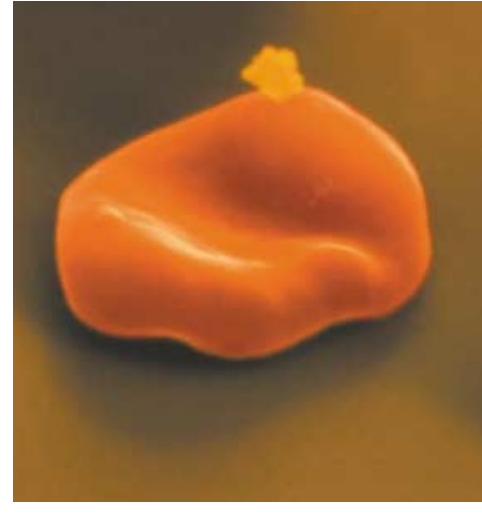

MALARIA

\section{Old parasites fight back}

Knowing thy enemy puts you in a stronger position to defeat it. This tactic is no more true in life than in combatting widespread and potentially deadly infections, such as those caused by the malaria parasite Plasmodium falciparum (see picture), which kills $\sim 2.7$ million people each year. Efforts to curb P. falciparum have met with relative success - but ultimately the effectivess of any drug or vaccine will depend largely on how old, and therefore how variable, this parasite really is. If, as some believe, the parasite suffered a severe bottleneck 3,000-5,000 years ago, then the existing parasite isolates would be too genetically homogeneous to develop resistance to drugs that are directed against it. Appealing though it might be, this view, which is known as the Malaria's Eve hypothesis, is also very controversial. Now, two investigations, both led by Xin-zhuan $\mathrm{Su}$, knock back this idea through an analysis of the largest ever genome sampling of $P$. falciparum. The genetic diversity of $P$. falciparum suggests that this parasite could be as much as 180,000 years old, and that, as feared, it rapidly develops resistance to anti-malarial drugs.

Different studies on the age of $P$. falciparum have given wildly different estimates. To settle the matter, Jianbing $\mathrm{Mu}$ and colleagues examined isolates from five divergent parasite clones for variation in SNPs and microsatellites in the coding and non-coding regions of 204 genes, all on chromosome 3 . The markers were valuable for constructing a molecular map of this chromosome, which can be used for future gene-mapping studies. More importantly for the issue at hand, however, was the level of genetic change observed in neutrally evolving sequences. There was no evidence of a severe recent bottleneck in the parasite's genetic history. Instead, the analysis revealed that the $P$. falciparum population is very ancient and diverse, and that it could have been present when humans first started migrating around the globe. The malaria parasite could therefore be as diverse as we are - and so pose a threat to existing vaccination strategies.

What does this mean for the parasite's ability to resist drugs? The results of the second study are rather disheartening. Wootton et al. analysed the variation in and around the $p f c r t$ gene, which confers resistance to chloroquine, a common anti-malarial drug. The level of diversity in and around this gene measured by genotyping microsatellite markers in 87 isolates - was noticeably higher in drug-sensitive than in drug-resistant strains, showing that the selective pressure of using drugs can decrease variability at specific loci. The authors further show that, instead of there being only two independently evolved chloroquine-resistant alleles, there are four, and that they are spreading quickly through the population.

It's unlikely that, on the basis of this work alone, malarial researchers will reach a consensus on the true age and genetic diversity of $P$. falciparum, and on the impact of this knowledge on public health measures. However, in an accompanying News and Views, Andrew Clark is optimistic that, through more thorough genetic monitoring and by creating common $P$. falciparum collections and databases, agreement shouldn't be too far off.

Tanita Casci

\section{(9) References and links} ORIGINAL RESEARCH PAPERS $\mathrm{Mu}$, J. et al

Chromosome-wide SNPs reveal an ancient origin for Plasmodium falciparum. Nature 418, 323-326 (2002) | Wootton, J. C. et al. Genetic diversity and chloroquine selective sweeps in Plasmodium

falciparum. Nature 418, 320-323 (2002) FURTHER READING Clark, A. G. Malaria variorum. Nature 418, 283-285 (2002)

\section{IN BRIEF}

\section{MOUSE MODELS}

Mice with truncated MeCP2 recapitulate many Rett syndrome features and display hyperacetylation of histone $\mathrm{H} 3$.

Shahbazian, M. D. et al. Neuron 35, 243-254 (2002)

How mutations in $M E C P 2$, a methyl-CpG-binding-protein gene, cause the neurological disorder Rett syndrome (RTT) is puzzling because its loss is predicted to cause genome-wide transcriptional de-repression. To investigate this, Shahbazian et al. generated a mouse model of RTT using a truncated Mecp2. Mutant tissues showed increased histone $\mathrm{H} 3$ acetylation, implicating Mecp2 in chromatin modification. How this affects gene expression to cause RTT remains unknown.

\section{EVOLUTION}

\section{Evolution of the gene network underlying wing} polyphenism in ants.

Abouheif, E. \& Wray, G. A. Science 297, 249-252 (2002)

Polyphenism - a genome's ability to produce alternative morphologies depending on environmental cues - is common in social insects. In ants, reproductive castes have wings, whereas sterile castes (workers and soldiers) don't. By looking at changes in gene expression, these authors determined that the gene networks that control wing development are interrupted in wingless ants. But where this block occurs varies in different species, despite a single evolutionary origin of wing polypheny, suggesting that the process is evolutionarily labile.

\section{TECHNOLOGY}

Cloned zebrafish by nuclear transfer from long-termcultured cells.

Lee, K. Y. et al. Nature Biotechnol. 20, 795-799 (2002)

Lee et al. now report the first cloning, by nuclear transfer, of a non-mammalian vertebrate - zebrafish. To achieve this, pronuclei were mechanically removed from mature eggs and replaced with donor nuclei, which here were marked with GFP and came from primary embryonic cells or from cultured zebrafish cells. Its $2 \%$ success rate means this technique will facilitate targeted genetic manipulation in zebrafish.

\section{EVO-DEVO}

Cloning and expression of Xenopus Prickle, an orthologue of a Drosophila planar polarity gene.

Wallingford, J. B. et al. Mech. Dev. 116, 183-186 (2002)

One nagging question in evo-devo concerns whether the same molecules underlie morphogenetic movements in vertebrates and invertebrates. Work in Drosophila showed that convergent movements involve the planar cell polarity (PCP) signalling pathway, which is controlled by the Prickle protein. Here, the authors have cloned the Xenopus homologue of Prickle (XPk). Its expression supports the role of PCP signalling in vertebrate morphogenesis, specifically during gastrulation and neurulation. 